\title{
Effects of Nintedanib in an Animal Model of Liver Fibrosis
}

\author{
Lutz Wollin $\mathbb{D}^{1}{ }^{1}$ Dieudonnée Togbe, ${ }^{2}$ and Bernhard Ryffel $^{3}$ \\ ${ }^{1}$ Immunology and Respiratory, Boehringer Ingelheim Pharma GmbH \& Co. KG, Biberach 88397, Germany \\ ${ }^{2}$ Research and Development Department, Artimmune, Orléans 45100, France \\ ${ }^{3}$ Molecular Immunology, INEM, UMR7355, CNRS and University Orléans, Orléans 45071, France
}

Correspondence should be addressed to Lutz Wollin; stefan-lutz.wollin@boehringer-ingelheim.com

Received 15 January 2020; Accepted 3 March 2020; Published 31 March 2020

Academic Editor: Nicholas Dunne

Copyright (c) 2020 Lutz Wollin et al. This is an open access article distributed under the Creative Commons Attribution License, which permits unrestricted use, distribution, and reproduction in any medium, provided the original work is properly cited.

\begin{abstract}
Systemic sclerosis can affect multiple internal organs, including the liver and lungs. Nintedanib, an antifibrotic approved for treatment of interstitial lung disease associated with systemic sclerosis, may have activity outside of the lungs. This study explored the effect of preventive and therapeutic nintedanib treatment in a 3-week carbon tetrachloride $\left(\mathrm{CCL}_{4}\right)$-induced $(500 \mathrm{mg} / \mathrm{kg} / \mathrm{day}$ twice weekly for 3 weeks) model of hepatic inflammation and fibrosis in C57Bl/ 6 mice (aged 8 weeks, $n=5$ per group). Mice also received nintedanib (30 or $60 \mathrm{mg} / \mathrm{kg} /$ day) either each day for 21 days (preventive treatment) or from day 7 or day 14 (therapeutic treatment). Preventive nintedanib treatment at both doses significantly reduced $\mathrm{CCL}_{4}$-induced increases in myeloperoxidase $(p<0.01)$, hepatic collagen $(p<0.001)$, and interleukin (IL) $6(p<0.01)$ in the liver. Nintedanib also significantly reduced hepatic necrosis $(p<0.01$ and $p<0.05)$, inflammation $(p<0.001$ and $p<0.05)$, fibrosis $(p<0.001$ and $p<$ $0.05)$ and IL- $\beta(p<0.05$ and $p<0.001)$ at both 30 and $60 \mathrm{mg} / \mathrm{kg} /$ day, respectively. Therapeutic treatment with nintedanib at 30 and $60 \mathrm{mg} / \mathrm{kg} /$ day significantly reduced $\mathrm{CCL}_{4}$-induced serum alanine aminotransferase from day $7(p<0.05$ and $p<0.001)$ and day $14(p<0.01$ and $p<0.05)$, respectively. Increases in tissue inhibitor of metalloproteinase- 1 were significantly reduced by nintedanib at $60 \mathrm{mg} / \mathrm{kg} /$ day from day 7 only $(p<0.001)$, and nintedanib completely blocked elevation of IL- 6 and IL- $1 \beta$ levels regardless of dose or start of treatment $(p<0.05-p<0.001)$. In both the preventive and therapeutic treatment schedules of the study, nintedanib treatment was beneficial in attenuating $\mathrm{CCL}_{4}$-induced pathology and reducing hepatic injury, inflammation, and fibrosis, demonstrating that nintedanib has antifibrotic and anti-inflammatory activity outside of the lungs.
\end{abstract}

\section{Introduction}

Nintedanib is a small-molecule tyrosine kinase inhibitor that targets the receptors of certain fibrosis-related kinases, including PDGF, FGF, VEGF, and transforming growth factor-beta $(\operatorname{TGF} \beta)[1,2]$. Nintedanib also inhibits tyrosine kinases of the Src family such as Src, Lck, and Lyn and CSF1R that are involved in inflammation, proliferation, and immunological activation [2].

Systemic sclerosis (SSc) is a chronic disease characterised by endothelial dysfunction, resulting in alterations of the microvasculature, immune dysregulation, fibroblast dysfunction, and subsequent fibrosis [3]. Due to the heterogeneity of the disease, SSc remains a major clinical challenge for both physicians and patients [4]. The clinical course is variable, but SSc can affect multiple internal organs, particularly early in the disease, including the lungs, liver, kidneys, and heart $[5,6]$.

Nintedanib is an antifibrotic treatment currently approved in the United States, Canada, and Japan to slow the rate of decline in pulmonary function in adults with interstitial lung disease (ILD) associated with SSc (SSc-ILD) and in many countries for idiopathic pulmonary fibrosis (IPF). The INPULSIS [7] and TOMORROW [8] trials demonstrated the beneficial effects of nintedanib in patients with IPF. A pooled analysis of data from both trials showed that nintedanib was associated with a reduction in the annual rate of decline in forced vital capacity (FVC), fewer acute exacerbations, and the preservation of health-related quality of life [9].

Although the clinical course of SSc-ILD can vary, many patients experience disease progression with a decline in 
Treatment schedule

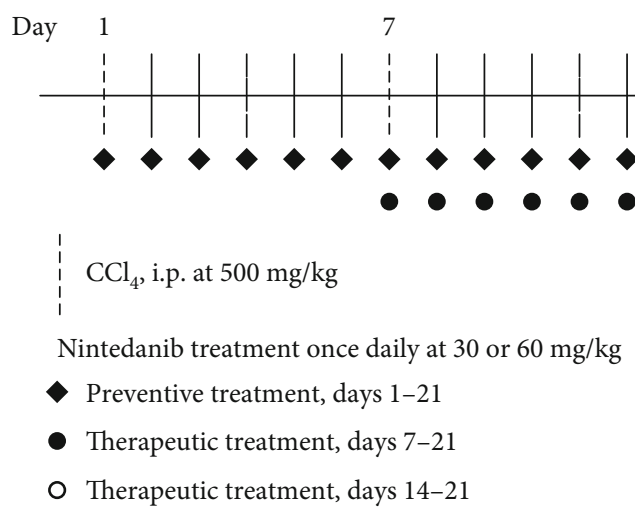

FIGURE 1: Treatment schedule in a mouse model of liver fibrosis. During the preventive phase, drug treatment started simultaneously with $\mathrm{CCL}_{4}$ injections from day 0 . In the therapeutic phase, drug treatment was administered on day 7 or day 14 of $\mathrm{CCL}_{4}$ injections. Mice received nintedanib 30 or $60 \mathrm{mg} / \mathrm{kg} /$ day once daily. $\mathrm{CCL}_{4}$ : carbon tetrachloride; i.p.: intraperitoneal.

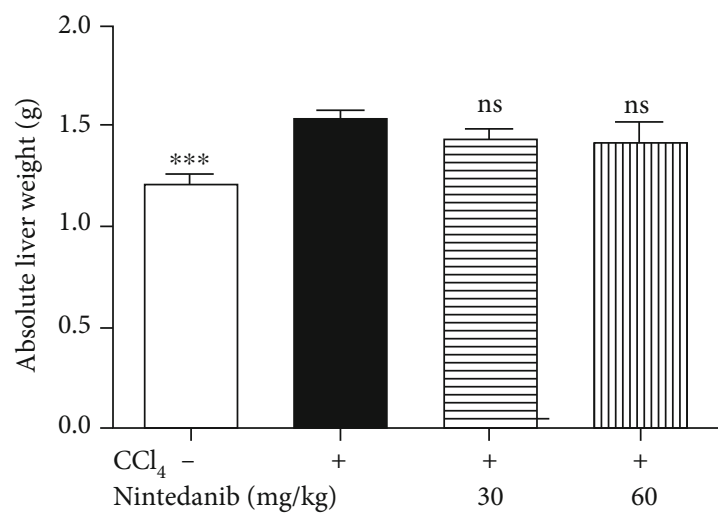

(a)

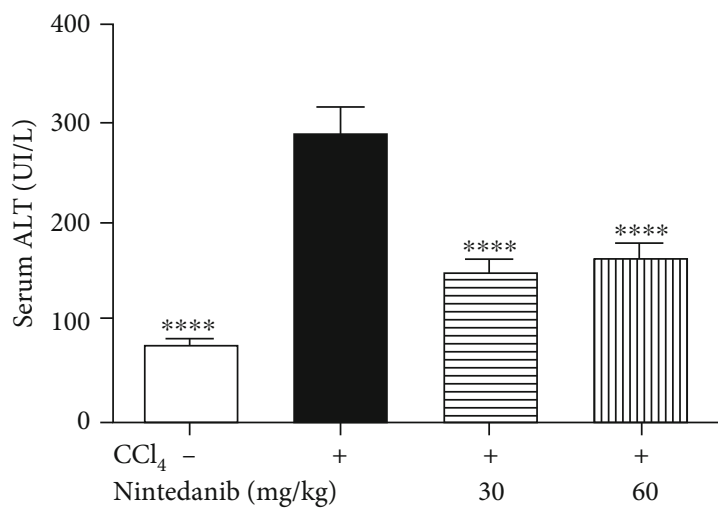

(c)

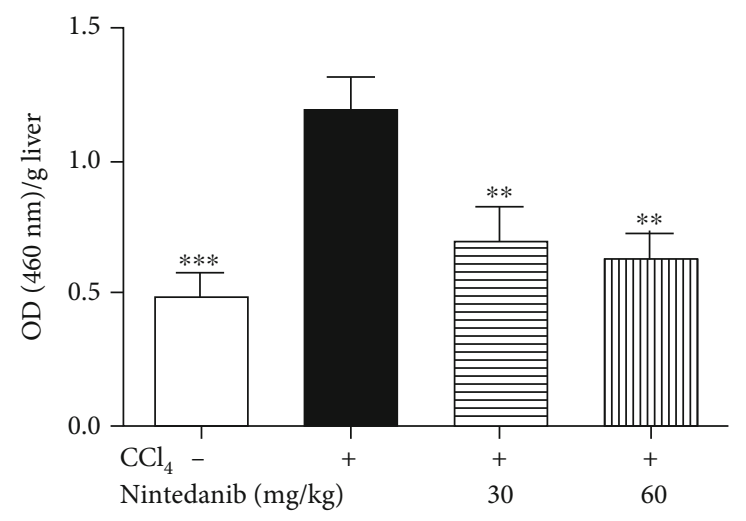

(b)

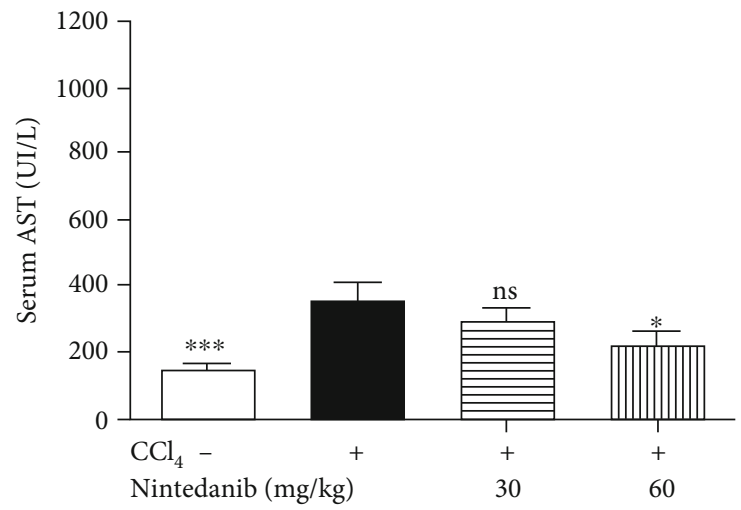

(d)

FIGURE 2: Effect of nintedanib on (a) liver weight and concentration in liver tissue of (b) myeloperoxidase (optical density), (c) serum alanine aminotransferase, and (d) serum aspartate aminotransferase during the preventive phase. Data are presented as mean \pm standard error of the mean; $n=5$ per group. Statistical significance is shown as: ${ }^{*} p<0.05,{ }^{* *} p<0.01,{ }^{* * *} p<0.001,{ }^{* * * *} p<0.0001$. ALT: alanine aminotransferase; ASP: aspartate aminotransferase; $\mathrm{CCL}_{4}$ : carbon tetrachloride; ns: not significant.

FVC associated with death [10,11]. In the SENSCIS trial, nintedanib reduced the annual rate of decline in FVC compared with placebo [12]. The INBUILD trial evaluated nintedanib in patients with ILDs that have a progressive fibrosing phenotype; a subset of patients in the study have ILD associated with a connective tissue disease (CTD), e.g., rheumatoid arthritis (RA). Nintedanib reduced the annual rate of decline in FVC in the overall population, as well as in subgroups of 


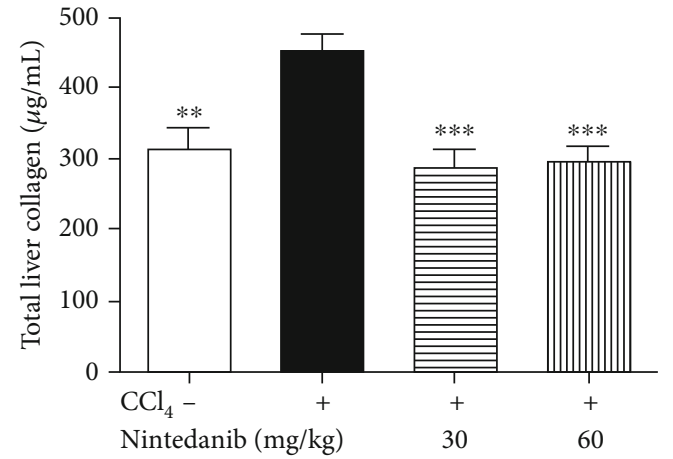

(a)

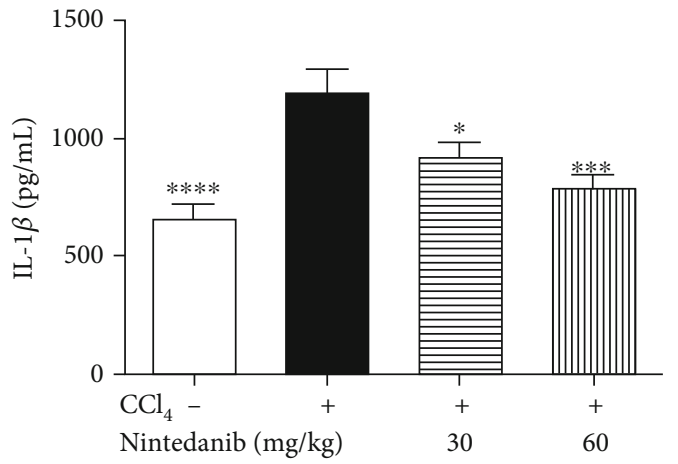

(c)

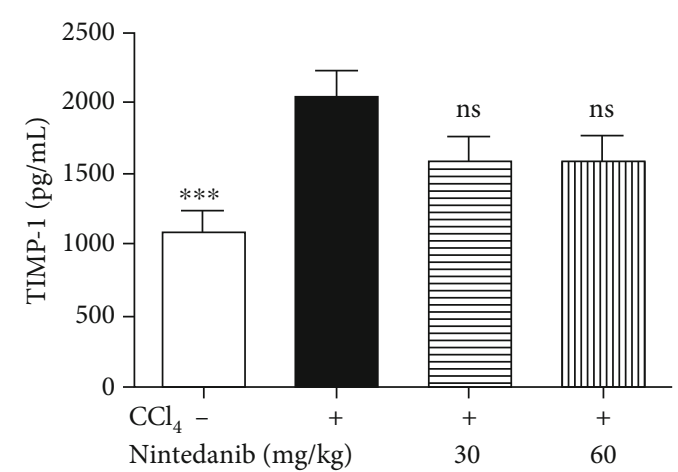

(b)

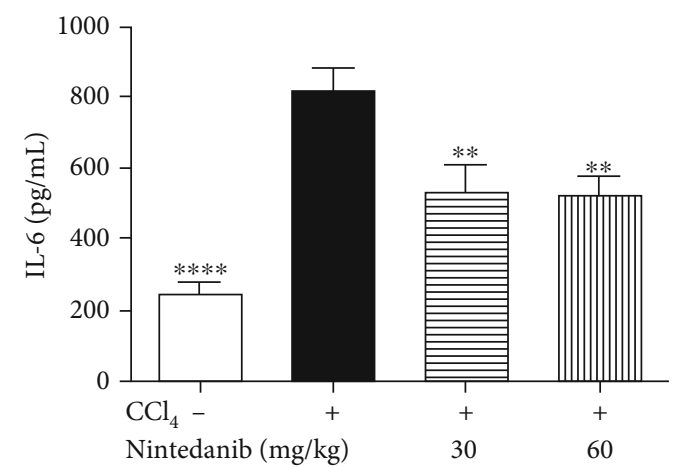

(d)

Figure 3: Effect of nintedanib on (a) total liver collagen and concentration in liver tissue of (b) TIMP-1, (c) IL-1 $\beta$, and (d) IL-6 during the preventive phase. Data are presented as mean \pm standard error of the mean; $n=5$ per group. Statistical significance is shown as ${ }^{*} p<0.05$, ${ }^{* *} p<0.01,{ }^{* * *} p<0.001,{ }^{* * * *} p<0.0001$. $\mathrm{CCL}_{4}$ : carbon tetrachloride; IL-1 $\beta$ : interleukin-1 $\beta$; IL-6: interleukin-6; ns: not significant; TIMP-1: tissue inhibitor of metalloproteinase.

patients with usual interstitial pneumonia-like or other fibrotic patterns [13]. Nintedanib has been well tolerated, with an acceptable safety profile and with diarrhoea the most common adverse event $[7,8,12]$.

In addition to being associated with pulmonary manifestations, CTDs may also have an effect on the liver, typically as biochemical changes with a cholestatic or hepatocellular pattern. In rare cases, patients with CTDs may develop progressive liver disease, including liver fibrosis [14]. Given that multiple organs can be affected in patients with CTD, as well as the known antifibrotic and anti-inflammatory action of nintedanib, we planned to explore what effect nintedanib might have on liver injury.

Hepatic injury by carbon tetrachloride $\left(\mathrm{CCL}_{4}\right)$ or ethanol causes inflammation, steatosis, and fibrosis that is toll-like receptor (TLR) 4 and TGF $\beta$-signalling dependent [1] and models human liver cirrhosis. Nintedanib, which reduces TGF $\beta$ and inflammatory mediators, may therefore have antifibrotic efficacy outside its known activity in the lungs. A previous preclinical acute study has shown antifibrotic effects of nintedanib in a 4-day $\mathrm{CCL}_{4}$ model of liver injury and fibrosis [15].

This study aimed to investigate whether nintedanib has antifibrotic activity outside of the lungs. The current study was undertaken to explore the effect of preventive and therapeutic nintedanib treatment in a 3 -week $\mathrm{CCL}_{4}$-induced animal model of liver inflammation and fibrosis.

\section{Materials and Methods}

2.1. $\mathrm{CCL}_{4}$-Induced Model of Liver Fibrosis. We used a $\mathrm{CCL}_{4}{ }^{-}$ induced model of hepatic inflammation and fibrosis in C57Bl/6 mice (Janvier, Le Genest-Saint-Isle, France). Mice were aged 8 weeks and caged in groups of five with ad libitum access to food and water. Animals received intraperitoneal $\mathrm{CCL}_{4}$ (Sigma-Aldrich, St. Louis, Missouri, United States) at $500 \mathrm{mg} / \mathrm{kg} /$ day twice weekly for 3 weeks to induce hepatic injury, inflammation, and fibrosis. Hepatic fibrosis was analysed at day 21 . This study was approved by the regional ethics committee (CL2007-021).

2.2. Study Design. The study included two parts: a preventive and a therapeutic protocol. In the preventive phase, nintedanib treatment started simultaneously with $\mathrm{CCL}_{4}$ injections from day 0 to evaluate the effect of nintedanib in preventing hepatic injury. In the therapeutic phase, drug treatment was started on day 7 or day 14 of $\mathrm{CCL}_{4}$ injections to evaluate the effect of nintedanib in treating already established hepatic injury and fibrosis.

2.3. Treatment Protocols. Nintedanib 30 and $60 \mathrm{mg} / \mathrm{kg} / \mathrm{day}$ (provided by Boehringer Ingelheim Pharma $\mathrm{GmbH} \& \mathrm{Co}$. $\mathrm{KG})$ was administered once daily by oral gavage. Administration volume was $10 \mathrm{~mL} / \mathrm{kg}$ body weight. In the preventive protocol (Figure 1), there were four treatment groups 


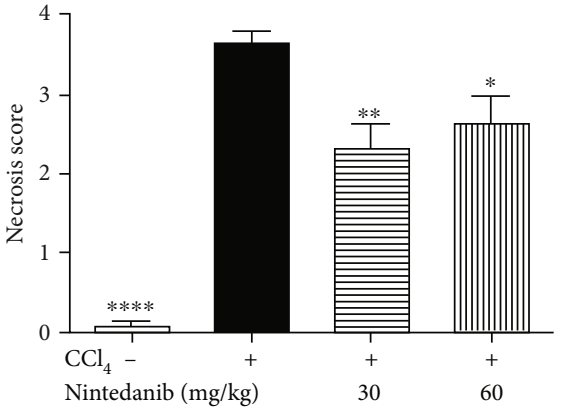

(a)

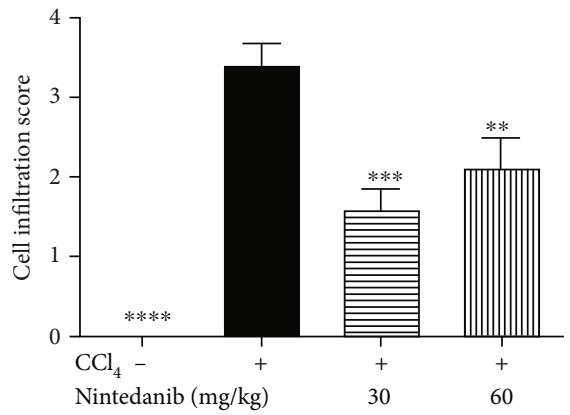

(b)

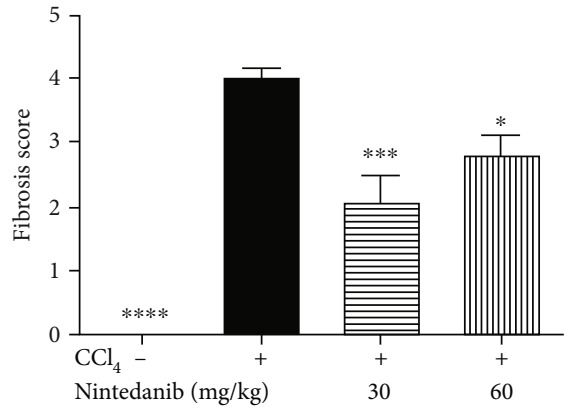

(c)

Figure 4: Effect of nintedanib on (a) liver necrosis, (b) liver inflammatory cell infiltration, and (c) liver fibrosis score during the preventive phase. Data are presented as mean \pm standard error of the mean; $n=5$ per group. Statistical significance is shown as ${ }^{*} p<0.05$, ${ }^{* *} p<0.01$, ${ }^{* * *} p<0.001,{ }^{* * * *} p<0.0001 . \mathrm{CCL}_{4}$ : carbon tetrachloride; ns: not significant.

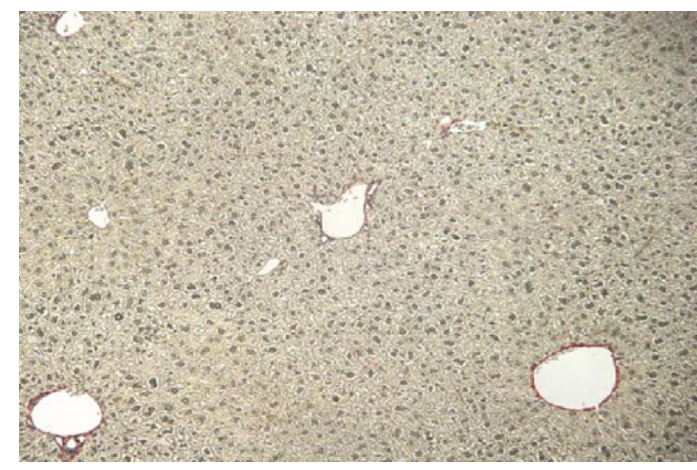

(a)

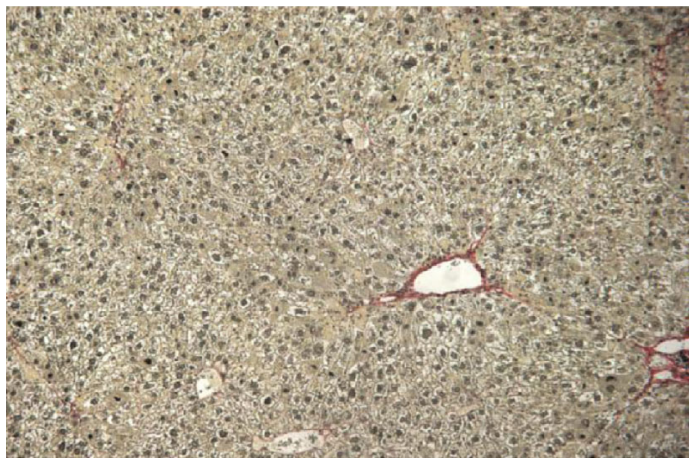

(c)

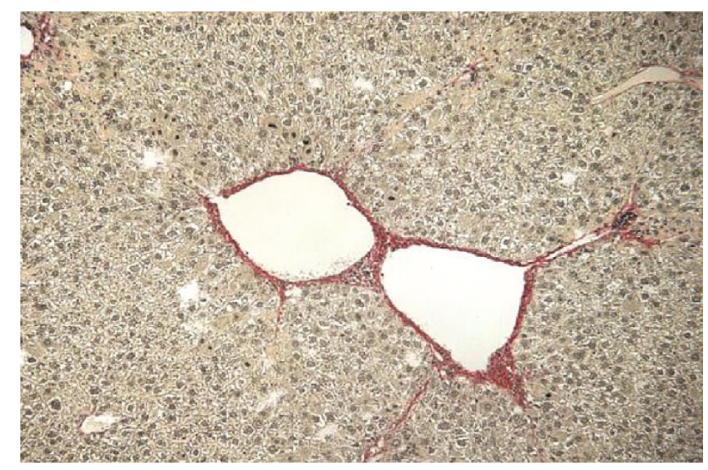

(b)

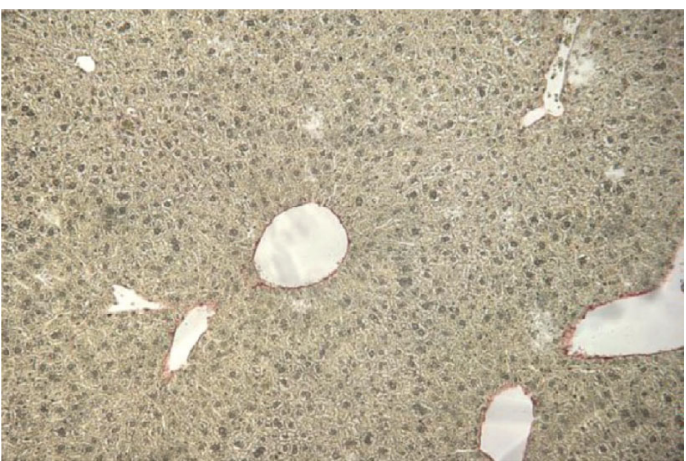

(d)

FIGURE 5: Representative histology slides stained with Sirius Red at 10-fold magnification during the preventive phase of (a) control without $\mathrm{CCL}_{4}$ stimulation, (b) control with $\mathrm{CCL}_{4}$ stimulation, (c) $\mathrm{CCL}_{4}$ and nintedanib at $30 \mathrm{mg} / \mathrm{kg} / \mathrm{day}$, (d) $\mathrm{CCL}_{4}$ and nintedanib $60 \mathrm{mg} / \mathrm{kg} / \mathrm{day}$. $\mathrm{CCL}_{4}$ : carbon tetrachloride.

( $n=10$ per group): control (corn oil); $\mathrm{CCL}_{4}$ and oral vehicle; $\mathrm{CCL}_{4}$ and nintedanib $30 \mathrm{mg} / \mathrm{kg} /$ day; and $\mathrm{CCL}_{4}$ and nintedanib $60 \mathrm{mg} / \mathrm{kg} / \mathrm{day}$. In the therapeutic protocol, there were six treatment groups ( $n=10$ per group): control (corn oil); $\mathrm{CCL}_{4}$ and oral vehicle; $\mathrm{CCL}_{4}$ and nintedanib at $30 \mathrm{mg} / \mathrm{kg} / \mathrm{day}$ starting at day 7; $\mathrm{CCL}_{4}$ and nintedanib $60 \mathrm{mg} / \mathrm{kg} /$ day starting at day $7 ; \mathrm{CCL}_{4}$ and nintedanib at $30 \mathrm{mg} / \mathrm{kg} /$ day starting at day 14; and $\mathrm{CCL}_{4}$ and nintedanib $60 \mathrm{mg} / \mathrm{kg} /$ day starting at day 14.

2.4. Analyses. Body weight and clinical signs were recorded daily. All other analyses were conducted on day 21. Mice were euthanised at day 21 of the study, and the macroscopic analysis of the liver and liver weight changes was recorded postmortem. Alanine aminotransferase (ALT) and aspartate aminotransferase (AST) were determined in serum. Left liver lobes were homogenised to determine myeloperoxidase (MPO) activity, total liver collagen concentration by Sircol $^{\mathrm{TM}}$ assay, interleukin (IL)-1 $\beta$, IL-6, tumour necrosis factor-alpha (TNF $\alpha$ ), and TGF $\beta$ by enzyme-linked immunosorbent assay. Right liver lobes were fixed for histology (haematoxylin and eosin and Sirius Red F 3B staining) for semiquantitative assessment of liver necrosis, inflammatory cell infiltration, and fibrosis on a scale of $0-5(0=$ least severe and $5=$ most 


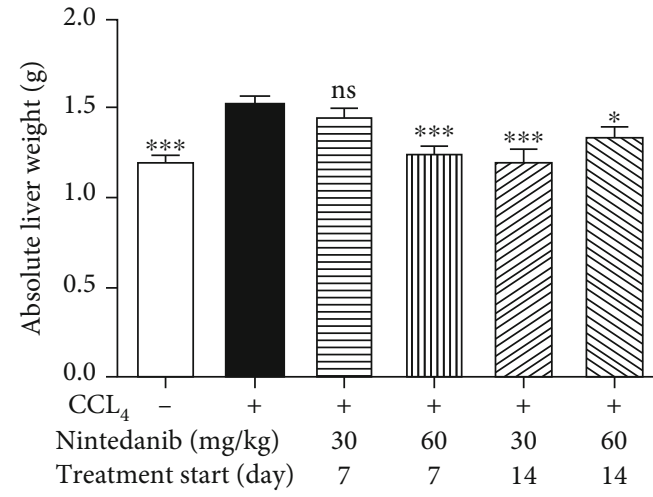

(a)

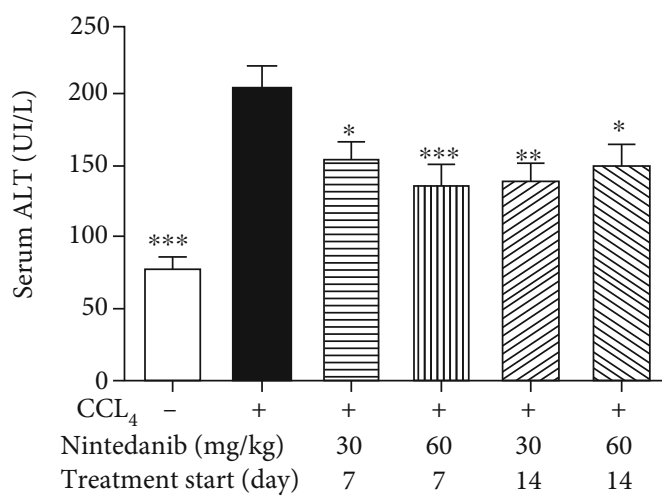

(c)

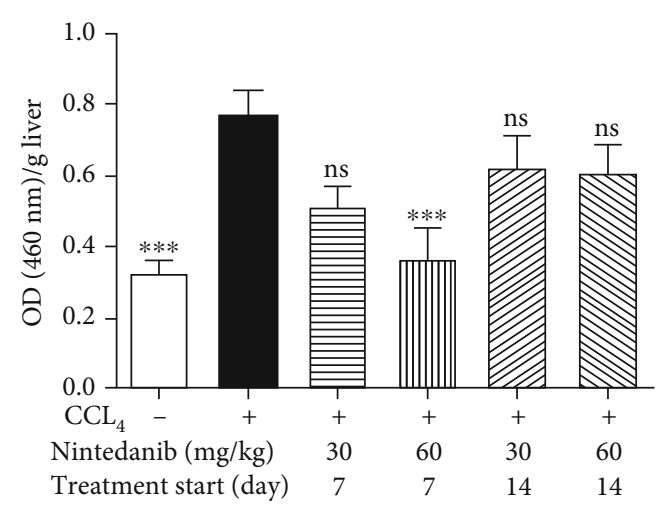

(b)

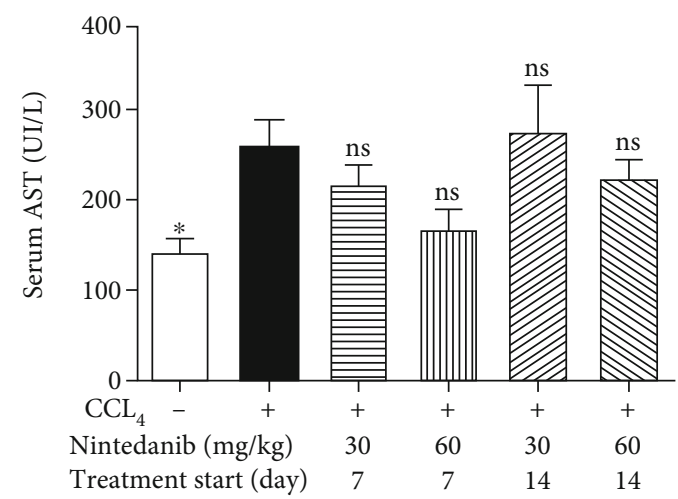

(d)

FIGURE 6: Effect of nintedanib on (a) liver weight and concentration in liver tissue of (b) myeloperoxidase, (c) alanine aminotransferase, and (d) aspartate aminotransferase during the preventive phase. Data are presented as mean \pm standard error of the mean; $n=5$ per group. Statistical significance is shown as ${ }^{*} p<0.05,{ }^{* *} p<0.01,{ }^{* * *} p<0.001$. ALT: alanine aminotransferase; ASP: aspartate aminotransferase; $\mathrm{CCL}_{4}$ : carbon tetrachloride; ns: not significant.

severe). Details of the methods can be found in Seki et al. [1] and Lisbonne et al. [16].

All data are presented as mean \pm standard error of the mean. Statistical differences between groups were analysed by one-way analysis of variance with subsequent Dunnett's multiple comparison test for all parametric data and KruskalWallis test for nonparametric data (GraphPad Prism 5.04). Statistical significance was accepted at $p<0.05$. All groups were tested against $\mathrm{CCL}_{4}$-treated positive control animals.

\section{Results}

3.1. Preventive Treatment. Nintedanib treatment resulted in a dose-dependent weak trend towards reduced body weight (<5\% at $60 \mathrm{mg} / \mathrm{kg} / \mathrm{day}$ ) compared with control or $\mathrm{CCL}_{4}$-only treated animals, with no statistical difference at day 21 (data not shown). Nintedanib had no effect on $\mathrm{CCL}_{4}$-induced liver weight gain (Figure 2(a)), and no other alterations of major organs were observed. Neutrophil recruitment into the liver was assessed by MPO activity determined in liver homogenate (Figure 2(b)). Nintedanib treatment with either 30 or $60 \mathrm{mg} / \mathrm{kg} /$ day significantly reduced MPO activity $(p<0.01)$. In mice treated with nintedanib, there was a significant reduction of $\mathrm{CCL}_{4}$-induced increase in ALT at 30 and $60 \mathrm{mg} / \mathrm{kg} / \mathrm{day}$ $(p<0.0001)$ and AST serum levels at $60 \mathrm{mg} / \mathrm{kg} / \mathrm{day}(p<0.05)$ (Figures 2(c) and 2(d)).

Nintedanib at both doses blocked $\mathrm{CCL}_{4}$-induced increase in hepatic collagen $(p<0.001)$ (Figure 3(a)). Nintedanib reduced tissue inhibitor of metalloproteinase (TIMP)-1, IL$1 \beta$, and IL- 6 in the liver (Figures $3(\mathrm{~b})-3(\mathrm{~d})$ ). This reduction was significant at both doses for IL-6 $(p<0.01)$ and for IL$1 \beta(30 \mathrm{mg} / \mathrm{kg} / \mathrm{day} ; p<0.05$ and $60 \mathrm{mg} / \mathrm{kg} / \mathrm{day} ; p<0.001)$. TNF $\alpha$, TGF $\beta$, and C-X-C motive ligand-1 (KC) levels were not affected by nintedanib administration (data not shown). Nintedanib significantly reduced liver necrosis $(p<0.01$ and $p<0.05)$, infiltration of inflammatory cells $(p<0.001$ and $p<0.01)$, and fibrosis $(p<0.001$ and $p<0.05)$ at both 30 and $60 \mathrm{mg} / \mathrm{kg} / \mathrm{day}$, respectively, as assessed by semiquantitative scoring of histology samples (Figures 4 and 5).

3.2. Therapeutic Treatment. Nintedanib treatment resulted in a weak trend towards reduced body weight $(<5 \%$ at $60 \mathrm{mg} / \mathrm{kg} /$ day) compared with control or $\mathrm{CCL}_{4}$-only treated animals from both days 7 and 14 (data not shown). Macroscopic examination of the major organs at necropsy on day 21 did not reveal any major organ alterations. Nintedanib significantly reduced $\mathrm{CCL}_{4}$-induced liver weight gain at both $30 \mathrm{mg} / \mathrm{kg} /$ day (from day $14 ; p<0.001$ ) and $60 \mathrm{mg} / \mathrm{kg} /$ day (from day 7 or $14 ; p<0.001$ and $p<0.05$, respectively) 


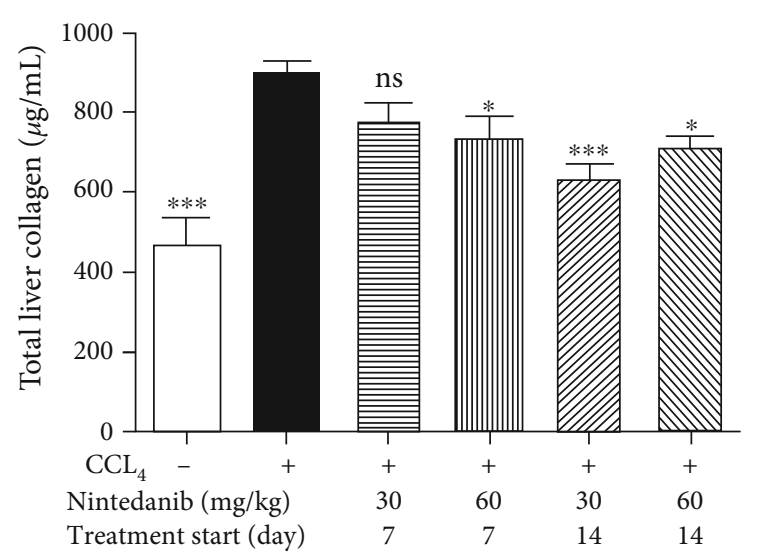

(a)

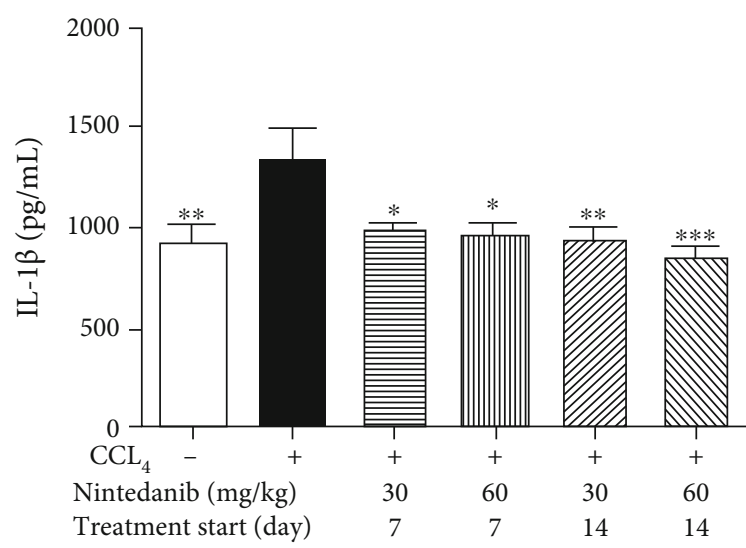

(c)

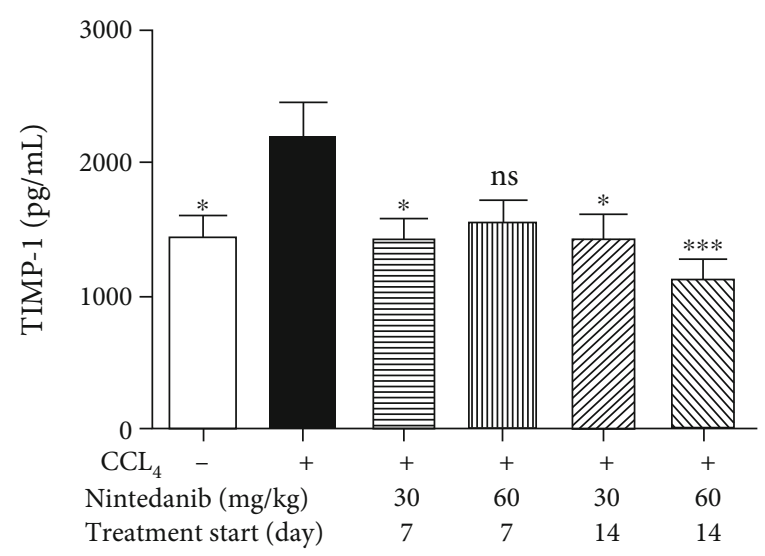

(b)

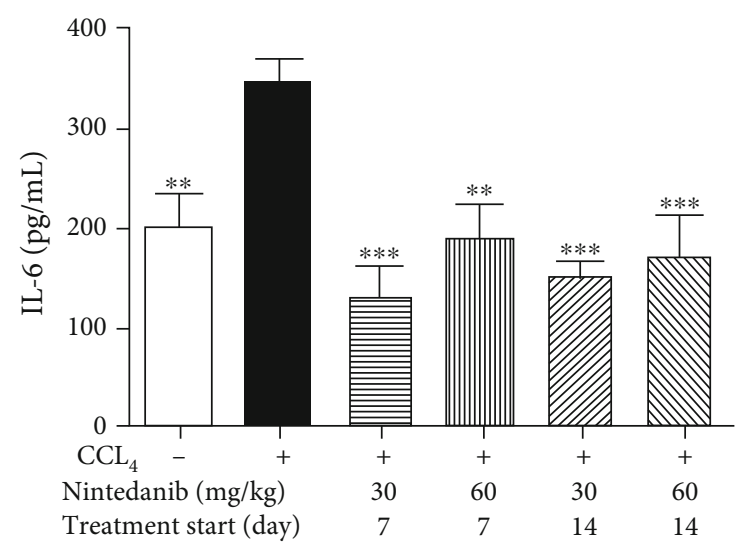

(d)

Figure 7: Effect of nintedanib on (a) total liver collagen and concentration in liver tissue of (b) TIMP-1, (c) IL-1 $\beta$, and (d) IL-6 during the therapeutic phase. Data are presented as mean \pm standard error of the mean; $n=5$ per group. Statistical significance is shown as ${ }^{*} p<0.05$, ${ }^{* *} p<0.01,{ }^{* * *} p<0.001 . \mathrm{CCL}_{4}$ : carbon tetrachloride; IL-1 $\beta$ : interleukin-1 $\beta$; IL-6: interleukin-6; ns: not significant; TIMP-1: tissue inhibitor of metalloproteinase.

(Figure 6(a)). There was a dose-dependent decrease in MPO if treatment started on day 7 only, reaching significance with $60 \mathrm{mg} / \mathrm{kg} /$ day $(p<0.001)$ (Figure $6(\mathrm{~b})$ ). ALT was significantly reduced following treatment with both doses of nintedanib, with treatment starting on either day $7(p<0.05$ and $p<0.001)$ or $14(p<0.01$ and $p<0.05)$ (Figure $6(c))$. There was a nonsignificant trend towards dose-dependent reduction in AST (Figure 6(d)).

Nintedanib significantly reduced the total liver collagen concentration at $30 \mathrm{mg} / \mathrm{kg} /$ day $(p<0.001$, from day 14) and at $60 \mathrm{mg} / \mathrm{kg} /$ day (from day 7 or $14, p<0.05$ ) (Figure $7(\mathrm{a})$ ). Increases in TIMP-1 were significantly reduced by nintedanib $30 \mathrm{mg} / \mathrm{kg} /$ day from days 7 and $14(p<0.05)$, and at $60 \mathrm{mg} / \mathrm{kg} /$ day from day 14 only $(p<0.001)$ (Figure $7(\mathrm{~b})$ ). Nintedanib completely blocked elevation of IL- 6 and IL- $1 \beta$ levels regardless of dose or start of treatment $(p<0.05$ $-p<0.001$ ) (Figures 7(c) and 7(d)). TNF $\alpha$, TGF $\beta$, and KC levels were not affected by nintedanib administration (data not shown). Nintedanib treatment at both doses significantly reduced liver necrosis, inflammation, and fibrosis, as assessed by semiquantitative histology, from day 7 only (Figures 8 (a)$8(\mathrm{c})$ ). The reduction in liver fibrosis appeared to be greater at $60 \mathrm{mg} / \mathrm{kg} / \mathrm{day}$ than at $30 \mathrm{mg} / \mathrm{kg} /$ day (Figure $8(\mathrm{c})$ ).

\section{Discussion}

In both the preventive and therapeutic treatment schedules of the study, nintedanib treatment attenuated $\mathrm{CCL}_{4}$-induced pathology and reduced hepatic injury, inflammation, and fibrosis.

In the preventive setting, there was a trend towards reduced ALT and AST in serum. Nintedanib treatment diminished neutrophil recruitment and reduced biochemical and inflammatory parameters suggestive of a beneficial effect on liver fibrosis. Both doses of nintedanib had similar preventive activities on most parameters explored but with no dose dependency.

Nintedanib treatment starting at day 7 was, in general, more effective than treatment starting at day 14; liver necrosis, inflammatory cell infiltration, and liver fibrosis, when assessed histologically, were only significantly reduced if treatment was started on day 7. This may have been because the pathology had already progressed too far or because treatment duration was too short to be effective when starting at day 14 (only 7 days of treatment). However, this study was not designed to describe statistically significant differences between treatments started at different time points. 


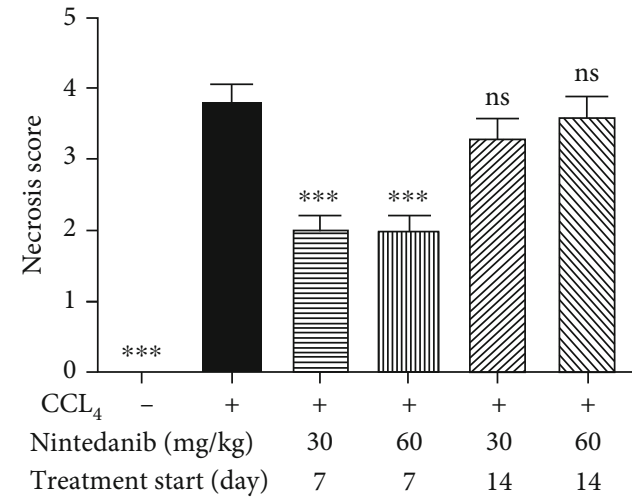

(a)

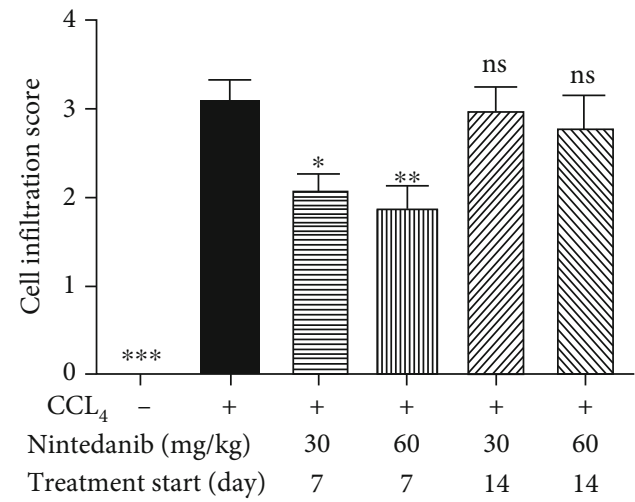

(b)

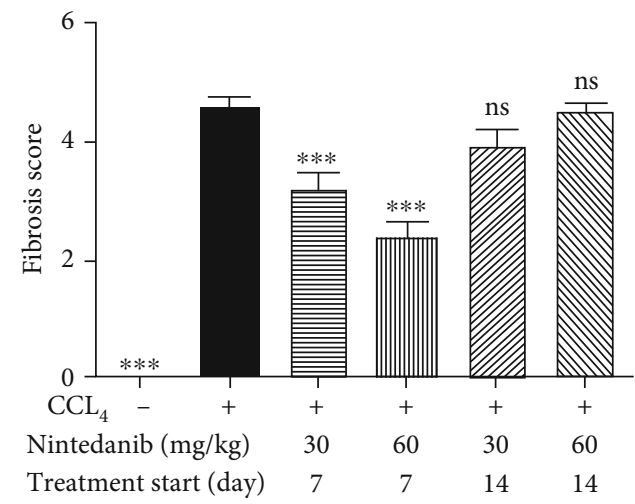

(c)

Figure 8: Effect of nintedanib on (a) liver necrosis, (b) liver inflammatory cell infiltration, and (c) liver fibrosis score during the therapeutic phase. Data are presented as mean \pm standard error of the mean; $n=5$ per group. Statistical significance is shown as ${ }^{*} p<0.05,{ }^{* *} p<0.01$, ${ }^{* * *} p<0.001 . \mathrm{CCL}_{4}$ : carbon tetrachloride; ns: not significant.

ALT/AST results were different from those seen in clinical trials, where elevated ALT/AST levels at least three times the upper limit of the normal range were observed in $4.9-13.0 \%$ of patients with lung fibrosis treated with nintedanib compared with placebo (up to $3.6 \%$ ), depending on the study $[7,8,12,13]$. In our mouse model, nintedanib did not increase ALT or AST serum levels. It may be that only patients with already impaired liver function before the start of clinical trials are prone to the increases in ALT and AST following nintedanib treatment.

Similar to our findings, another preclinical model of $\mathrm{CCL}_{4}$-induced liver fibrosis found that nintedanib significantly attenuated collagen accumulation and hepatic stellate cell activation, inhibiting intrahepatic inflammation and angiogenesis [15]. In a choline-deficient, L-amino aciddefined, high-fat diet-fed mouse model of nonalcoholic steatohepatitis, nintedanib also showed anti-inflammatory and antifibrotic effects [17].

Biochemical changes in the liver are common in patients with CTD. In rare cases, patients with CTDs may develop progressive liver disease, including liver fibrosis [14]. Liver involvement of this type is typically attributed to (coexisting) primary liver diseases (e.g., fatty liver disease, viral hepatitis, primary biliary cirrhosis, autoimmune hepatitis, and drug-related liver toxicity), rather than the CTD itself. Drug-induced liver injury is frequent with certain CTDs, particularly with the use of methotrexate in patients with RA, and may lead to progressive fibrosis and cirrhosis [18]. The results of the current study highlight the potential of nintedanib to reduce liver necrosis, inflammation, and fibrosis. As such, using nintedanib for the treatment of ILD in patients with CTDs, including SSc-ILD, may have antifibrotic effects in organs other than the lungs.

Nintedanib reduced and blocked $\mathrm{CCL}_{4}$-induced elevated hepatic IL- 6 in both the preventive and therapeutic phases of the study. Similar results were obtained in a mouse model of silica-induced lung fibrosis, where nintedanib reduced the IL-6 concentration in lung tissue [19]. Anti-IL-6 antibody therapy is used to treat RA-ILD, although evidence is controversial, with some studies showing beneficial effects and others finding ILD occurrence or exacerbation following tocilizumab treatment $[20,21]$. Interestingly, imatinib mesylate, another kinase inhibitor that has shown antifibrotic effects in the liver in preclinical studies, has been shown to increase serum IL-6 levels in conjunction with liver regeneration [22]. The current study shows a potential systemic effect of nintedanib on IL-6, indicating that further investigation of nintedanib in RA-ILD may be warranted.

However, there are limitations with this study, including those normally associated with animal models of disease. The $\mathrm{CCL}_{4}$ model is also more a model of acute liver injury leading to fibrosis and does not directly correspond to the clinical 
situation where patients normally present with chronic fibrosis [15]. However, it provides a good indication (along with other animal models of fibrosis discussed above) that nintedanib has antifibrotic activity outside of the lungs.

\section{Conclusions}

In conclusion, nintedanib demonstrated antifibrotic and anti-inflammatory activity, showing therapeutic potential in this experimental mouse model of $\mathrm{CCL}_{4}$-induced hepatic fibrosis.

\section{Data Availability}

To ensure independent interpretation of clinical study results, Boehringer Ingelheim grants all external authors access to all relevant material, including participant-level clinical study data, and relevant material as needed by them to fulfil their role and obligations as authors under the International Committee of Medical Journal Editors criteria. Furthermore, clinical study documents (e.g. study report, study protocol, statistical analysis plan) and participant clinical study data are available to be shared after publication of the primary manuscript in a peer-reviewed journal, and if regulatory activities are complete and other criteria are met per the BI Policy on Transparency and Publication of Clinical Study Data: https://trials.boehringer-ingelheim.com/ transparency_policy.html. Prior to providing access, documents will be examined, and, if necessary, redacted and the data will be de-identified, to protect the personal data of study participants and personnel, and to respect the boundaries of the informed consent of the study participants. Clinical Study Reports and Related Clinical Documents can be requested via this link: https://rials.boehringer-ingelheim .com/trial_results/clinical_submission_documents.html. All such requests will be governed by a Document Sharing Agreement. Bona fide, qualified scientific and medical researchers may request access to de-identified, analysable participant clinical study data with corresponding documentation describing the structure and content of the data sets. Upon approval, and governed by a Data Sharing Agreement, data are shared in a secured data-access system for a limited period of 1 year, which may be extended upon request. Researchers should use https://clinicalstudydatarequest.com to request access to study data.

\section{Conflicts of Interest}

Lutz Wollin is an employee of Boehringer Ingelheim Pharma $\mathrm{GmbH} \& \mathrm{Co}$. KG, who developed and market nintedanib. Dieudonnée Togbe is an employee of ArtImmune.

\section{Acknowledgments}

The authors would like to thank Isabelle Maillet of Immunologie Moléculaire, CNRS and University Orléans for her technical contribution to the study. The authors meet criteria for authorship as recommended by the International Committee of Medical Journal Editors (ICMJE). The authors received no direct compensation related to the development of the manuscript. Writing, editorial support, and formatting assistance were provided by Helen Keyworth, PhD, of Nucleus Global, which was contracted and funded by Boehringer Ingelheim International $\mathrm{GmbH}$ (BI). BI was given the opportunity to review the manuscript for medical and scientific accuracy as well as intellectual property considerations.

\section{References}

[1] E. Seki, S. de Minicis, C. H. Österreicher et al., "TLR4 enhances TGF- $\beta$ signaling and hepatic fibrosis," Nature Medicine, vol. 13, no. 11, pp. 1324-1332, 2007.

[2] F. Hilberg, G. J. Roth, M. Krssak et al., "BIBF 1120: triple angiokinase inhibitor with sustained receptor blockade and good antitumor efficacy," Cancer Research, vol. 68, no. 12, pp. 4774-4782, 2008.

[3] J. Varga, M. Trojanowska, and M. Kuwana, "Pathogenesis of systemic sclerosis: recent insights of molecular and cellular mechanisms and therapeutic opportunities," Journal of Scleroderma and Related Disorders, vol. 2, no. 3, pp. 137-152, 2017.

[4] C. P. Denton and D. Khanna, "Systemic sclerosis," Lancet, vol. 390, no. 10103, pp. 1685-1699, 2017.

[5] V. K. Jaeger, E. G. Wirz, Y. Allanore et al., "Incidences and risk factors of organ manifestations in the early course of systemic sclerosis: a longitudinal EUSTAR study," PLoS One, vol. 11, no. 10, article e0163894, 2016.

[6] T. M. Frech and D. Mar, "Gastrointestinal and hepatic disease in systemic sclerosis," Rheumatic Diseases Clinics of North America, vol. 44, no. 1, pp. 15-28, 2018.

[7] L. Richeldi, R. M. du Bois, G. Raghu et al., "Efficacy and safety of nintedanib in idiopathic pulmonary fibrosis," The New England Journal of Medicine, vol. 370, no. 22, pp. 2071-2082, 2014.

[8] L. Richeldi, U. Costabel, M. Selman et al., "Efficacy of a tyrosine kinase inhibitor in idiopathic pulmonary fibrosis," The New England Journal of Medicine, vol. 365, no. 12, pp. 10791087, 2011.

[9] L. Richeldi, V. Cottin, R. M. du Bois et al., "Nintedanib in patients with idiopathic pulmonary fibrosis: Combined evidence from the TOMORROW and INPULSIS ${ }^{\circledast}$ trials," Respiratory Medicine, vol. 113, pp. 74-79, 2016.

[10] N. S. Goh, S. R. Desai, S. Veeraraghavan et al., "Interstitial lung disease in systemic sclerosis: a simple staging system," American Journal of Respiratory and Critical Care Medicine, vol. 177, no. 11, pp. 1248-1254, 2008.

[11] N. S. Goh, R. K. Hoyles, C. P. Denton et al., "Short-term pulmonary function trends are predictive of mortality in interstitial lung disease associated with systemic sclerosis," Arthritis \& Rhematology, vol. 69, no. 8, pp. 1670-1678, 2017.

[12] O. Distler, K. B. Highland, M. Gahlemann et al., "Nintedanib for systemic sclerosis-associated interstitial lung disease," The New England Journal of Medicine, vol. 380, no. 26, pp. 2518 2528, 2019.

[13] K. R. Flaherty, A. U. Wells, V. Cottin et al., "Nintedanib in progressive fibrosing interstitial lung diseases," The New England Journal of Medicine, vol. 381, no. 18, pp. 1718-1727, 2019.

[14] A. A. Chentoufi, Y. A. Serov, M. Alazmi, and K. Baba, "Immune components of liver damage associated with connective tissue diseases," Journal of Clinical and Translational Hepatology, vol. 2, no. 1, pp. 37-44, 2014. 
[15] B. Ozturk Akcora, G. Storm, J. Prakash, and R. Bansal, "Tyrosine kinase inhibitor BIBF1120 ameliorates inflammation, angiogenesis and fibrosis in CCl4-induced liver fibrogenesis mouse model," Scientific Reports, vol. 7, p. 44545, 2017.

[16] M. Lisbonne, A. L'Helgoualc'h, G. Nauwelaers et al., "Invariant natural killer T-cell-deficient mice display increased $\mathrm{CCl}_{4}$ induced hepatitis associated with CXCL1 over-expression and neutrophil infiltration," European Journal of Immunology, vol. 41, no. 6, pp. 1720-1732, 2011.

[17] W. Susutlertpanya, H. Wakuda, N. Otani et al., "Histological evaluation of nintedanib in non-alcoholic steatohepatitis mice," Life Sciences, vol. 228, pp. 251-257, 2019.

[18] R. Conway and J. J. Carey, "Risk of liver disease in methotrexate treated patients," World Journal of Hepatology, vol. 9, no. 26, pp. 1092-1100, 2017.

[19] L. Wollin, I. Maillet, V. Quesniaux, A. Holweg, and B. Ryffel, "Antifibrotic and anti-inflammatory activity of the tyrosine kinase inhibitor nintedanib in experimental models of lung fibrosis," The Journal of Pharmacology and Experimental Therapeutics, vol. 349, no. 2, pp. 209-220, 2014.

[20] M. Shaw, B. F. Collins, L. A. Ho, and G. Raghu, "Rheumatoid arthritis-associated lung disease," European Respiratory Review, vol. 24, no. 135, pp. 1-16, 2015.

[21] A. Rubbert-Roth, D. E. Furst, J. M. Nebesky, A. Jin, and E. Berber, "A review of recent advances using tocilizumab in the treatment of rheumatic diseases," Rheumatology and Therapy, vol. 5, no. 1, pp. 21-42, 2018.

[22] C. S. Kuo and L. R. Young, "Interstitial lung disease in children," Current Opinion in Pediatrics, vol. 26, no. 3, pp. 320-327, 2014. 